\title{
Materiales didácticos para la enseñanza de habilidades emocionales y sociales en alumnado con TEA: una investigación colaborativa en educación Primaria y Secundaria ${ }^{1}$
}

Didactic Materials for the Teaching of Social and Emotional Skills in Pupils with ASD: A Collaborative Research in Primary and Secondary Education

Josefina Lozano Martínez

lozanoma@um.es

Maria del Carmen Cerezo Máiquez

mcarmen.cerezo@um.es

Irina Sherezade Castillo Reche

irinasherezade.castillo@um.es

Universidad de Murcia, Murcia, España

\section{Resumen:}

En este artículo se presentan los resultados de una investigación que ha conllevado la implementación de un programa de inter-

\section{Abstract:}

This article reports on an investigation resulting from the implementation of an intervention program for the development of

1 Esta investigación forma parte del proyecto LA COLABORACIÓN ESCUELA-FAMILIA ANTE LA ENSEÑANZA DE COMPETENCIAS EMOCIONALES Y SOCIALES EN ALUMNADO CON TRASTORNOS DEL ESPECTRO AUTISTA seleccionado en los Proyectos conjuntos de investigación e innovación educativa, en el marco del programa III del convenio de cooperación en materia de formación inicial y permanente del profesorado que ejerce en los niveles anteriores a la universidad, suscrito con la Comunidad Autónoma de la Región de Murcia (convocatoria R-28/2013, de 10 de enero).

2 Dirección para correspondencia (correspondence address):

Josefina Lozano Martínez. Dpto. de Didáctica y Organización Escolar. Facultad de Educación. Universidad de Murcia. Campus de Espinardo, s/n. 30100 Murcia (España). 
Materiales didácticos para la enseñanza de habilidades emocionales y sociales en alumnado con TEA: una investigación colaborativa en educación Primaria y Secundaria Josefina lozano Martínez, Maria del Carmen Cerezo Málquez e Irina Sherezade Castillo RECHE

vención para el desarrollo de habilidades emocionales y sociales en alumnado con Trastornos del Espectro Autista (TEA), desde la colaboración familia, escuela y universidad. Para ello, y desde un estudio de casos múltiple se implementó dicho programa con seis alumnos de edades comprendidas entre los 9 y 17 años valorados con dicho trastorno y escolarizados en Educación Primaria y Secundaria. Se utilizó un diseño de grupo único con pretestpostest. Así, antes del proceso de enseñanza el alumnado fue evaluado con relación a sus niveles de habilidades emocionales y sociales. Esta evaluación inicial pretendía situar el punto de partida de cada uno de los alumnos para desarrollar el proceso de enseñanza y aprendizaje, el cual se extendió durante dos sesiones por semana, en el centro educativo y en el hogar, de 45 minutos cada una a lo largo de un curso académico. Tras el proceso de intervención educativa, cada uno de los alumnos fue nuevamente evaluado. El programa de intervención contempla unos materiales didácticos diseñados para fomentar las habilidades emocionales y sociales que han sido implementados a través de un trabajo de colaboración entre padres, docente y equipo investigador universitario.

Los resultados muestran que, tras el proceso de intervención educativa, los alumnos participantes mejoraron su rendimiento en habilidades emocionales y sociales, y que el desarrollo de métodos colaborativos incide de forma positiva sobre dichos procesos de enseñanza.

\section{Palabras clave:}

Trastorno del espectro autista; habilidades emocionales y sociales; materiales didácticos y colaboración familia, escuela y universidad. social and emotional skills in pupils with Autism Spectrum Disorders (ASD). The study implied the collaboration among families, schools and a university. This program, which implied the study of multiple cases, was implemented with six pupils between the ages of 9 and 17 years who had been diagnosed with the disorder and were enrolled in primary and secondary education. We used a one-group design with a pretest-posttest design. So, before the process of teaching, the students were evaluated regarding their levels of emotional and social skills. This initial assessment was intended to establish the starting point for each of the students before the teaching/ learning process started-it consisted of two 45 minute sessions per week, one at school and the other one at home, over one academic year. After the educational intervention process, each of the students was again assessed. The intervention program provided a few materials designed to foster children's emotional and social skills. The materials were implemented through the collaboration among parents, school teachers and the university research team.

The results show that, after the educational intervention, participating students improved their performance in social and emotional skills, and the development of collaborative methods has a positive effect on these teaching processes.

\section{Key words:}

Autism spectrum disorder; emotional and social skills; materials and collaboration family, school and university.

\section{Résumé:}

Dans cet article nous présenterons les résultats d'une enquête qui a entrâné la mise en œuvre d'un programme d'intervention pour le développement des compétences psychologiques et sociales auprès d'élèves souffrant de troubles du spectre Autiste (TEA). Nous avons effectué un travail de collaboration entre la famille, l'école et l'université. Le tout, en partant d'une étude de cas multiples. Ce programme a été réalisé auprès de six élèves âgés de 9 à 17 ans et scolarisés dans l'enseignement primaire et secondaire. Pour cela, nous avons utilisé une conception de groupe unique avec pretest-postest. De cette manière, le processus d'enseignement-apprentissage des étudiants a été évalué par rapport à leurs niveaux de compétences psychologiques et sociales. Cette évaluation 
Materiales didácticos para la enseñanza de habilidades emocionales y sociales en alumnado con TEA: una investigación colaborativa en educación Primaria y Secundaria

josefina lozano Martínez, Maria del Carmen Cerezo Máiquez e Irina Sherezade Castillo

RECHE

initiale visait à placer le point de départ de chacun des élèves afin de développer ledit processus. Ce travail a été mis en place au cours de deux séances de 45 minutes par semaine, dans le centre éducatif et dans le foyer, tout au long de l'année universitaire. Après le processus d'intervention éducative, chaque élève a de nouveau été évalué. Le programme d'intervention prévoit des matériels didactiques conçus pour promouvoir les compétences psychologiques et sociales. Ils ont été mis en œuvre grâce à un travail de collaboration entre parents et enseignants, ainsi qu'un matériel chercheur universitaire.

Les résultats montrent que suite au processus d'intervention éducative, les élèves participants ont amélioré leur rendement en compétences psychologiques et sociales. De plus, le développement de méthodes collaboratives a eu une influence positive sur ces processus d'enseignement.

\section{Mots clés:}

Trouble du spectre autiste; compétences psychologiques et sociales; matériels didactiques et collaboration, école et université.

Fecha de recepción: 16-5-2017

Fecha de aceptación: 14-9-2017

\section{Introducción}

El autismo se define como un trastorno del desarrollo, muy complejo y heterogéneo, que presenta alteraciones, en mayor o menor grado, en las capacidades de reconocimiento social, comunicación social, imaginación y comprensión social y la presencia de patrones repetitivos de la actividad (Wing, 1988). Las personas con Trastorno del Espectro Autista (TEA) pueden presentar dificultad para la atribución de emociones y creencias que incide negativamente sobre sus capacidades de reconocimiento social. En esta investigación se plantea, como objetivo de estudio, el desarrollo de un proceso de enseñanza-aprendizaje para ayudar a alumnos con TEA a fomentar la capacidad de comprensión de emociones y creencias como un medio de aprendizaje y mejora de otras habilidades no enseñadas explícitamente, como las habilidades básicas de relación social, referencia conjunta y capacidad intersubjetiva. Hay estudios que informan de la posibilidad de que personas con TEA aprendan a realizar tareas de comprensión de emociones y creencias (Hadwin, Baron-Cohen, Howlin y Hill, 1996), sin embargo, no se ha hallado que tales aprendizajes se extrapolen a sus habilidades cotidianas de interacción y comunicación social, es decir que sean capaces de generalizar sus aprendizajes en todos los contextos. De ahí que nos preguntemos en el presente estudio si es posible la generalización de los aprendizajes realizados en contextos naturales (escuela y hogar) 
desde la colaboración de la familia-la escuela y el equipo universitario. Para ello, se ha utilizado un programa de intervención educativa que recoge distintos materiales didácticos, diseñados para este fin, que se implementarán en el contexto escolar y familiar desde el trabajo conjunto de ambas instituciones.

\section{Marco teórico}

El concepto clásico de autismo ha variado mucho desde su definición original por primera vez en 1943, por Leo Kanner, hasta nuestros días con la publicación del nuevo DSM-5 (Asociación de Psiquiatría Americana -APA-, 2013, 2014). Kanner (1943) realizó una primera descripción de lo que denominó "Autismo Infantil Precoz", identificándolo como una patología caracterizada por una extrema soledad, deseo obsesivo de que el ambiente no cambie, hipersensibilidad a los estímulos, así como retraso o alteración en la adquisición del habla y del lenguaje, entre otros. Hans Asperger, además, describe por primera vez, en 1944, las características de un grupo de niños muy similares a las descritas por Kanner, pero con mejor funcionamiento cognoscitivo, lo que identificó como un trastorno que produce dificultades considerables de integración social.

En la actualidad se habla de un continuo por lo que se prefiere usar el término Trastornos del Espectro del Autismo (TEA). En la nueva versión del DSM-5 (APA, 2013) se reúne bajo el concepto de TEA, lo que en versión anterior se contemplaba como trastorno autista, el síndrome de Asperger, el trastorno desintegrativo infantil y los trastornos no especificados del desarrollo. Esta categoría, además, agrupa como síntomas esenciales las deficiencias persistentes en la comunicación social y en la interacción social en diversos contextos y los patrones restrictivos y repetitivos de comportamiento, intereses o actividades.

Los síntomas, hasta ahora descritos, pueden ser desde leves hasta severos, por lo que existen distintos niveles, y hablamos, por consiguiente, de espectro (Rivière, 2002). Por ende, en el manual DSM-5 se establecen tres niveles de afectación que varían en función de la intensidad del apoyo que necesitan. De ahí su diversidad que nos lleva a reconocer que no hay dos personas con TEA idénticos (Lozano y Alcaraz, 2010). Respecto a las causas, a pesar de todos los avances en neurociencia y de los métodos genéticos, no se ha podido establecer un modelo que 
Materiales didácticos para la enseñanza de habilidades emocionales y sociales en alumnado con TEA: una investigación colaborativa en educación Primaria y Secundaria

Josefina lozano Martínez, Maria del Carmen Cerezo Máiquez e Irina Sherezade Castillo

RECHE

explique la etiología y fisiopatología de los TEA (García-Gómez, Risco, Rubio, Guerrero y García-Peña, 2014).

\section{Programas de intervención y proceso de enseñanza-aprendizaje para el desarrollo de habilidades emocionales y sociales}

Una de las mayores dificultades presentadas por las personas con TEA es el desarrollo de habilidades emocionales y sociales, careciendo por tanto de capacidades para entender lo que los demás piensan o sienten, interpretar las emociones que el resto de personas manifiestan, etc. Esos déficits que poseen las personas con TEA con relación a la teoría de la mente están asociados a las dificultades que presentan en la identificación y comprensión de estados emocionales, así como con el reconocimiento de mentiras e ironías (Blacher y Howell, 2007).

En la actualidad son diversas las investigaciones halladas con relación al desarrollo de la competencia emocional y/o social, en las que se constata una mejora en la identificación de emociones o la cognición social tras la implementación de programas dirigidos a ello (Bauminger, 2007; Bernard-Ripoll, 2007; Golan y Baron-Cohen, 2006; Golan, Ashwin, Granader, McClintock, Day, Leggett y Baron-Cohen, 2010; Howlin, 2008; Ojea, 2010; Tanaka, Wolf, Klaiman, Koenig, Cockburn, Herlihy y Schultz., 2010). Sin embargo, la dificultad mayor desde los resultados obtenidos en las diferentes investigaciones descritas resulta de la generalización de dichos aprendizajes. Por ello, en el presente estudio nos hemos planteado favorecer un proceso de enseñanza-aprendizaje en contextos naturales y desde la colaboración entre familias, docentes y equipo universitario para potenciar, de ese modo, dicha generalización.

En la búsqueda del logro de dicha generalización de los aprendizajes de un contexto a otro, hemos tenido en cuenta los resultados de estudios previos tanto en las dificultades que presentan el alumnado con TEA en la atribución de emociones como en la interacción social.

Así, en distintos estudios relacionados con la gran dificultad que supone para las personas con trastorno del espectro autista las tareas encaminadas a la atribución de emociones, se observaron anomalías ante la imitación de expresiones faciales emocionales (Loveland, Tunali-Kotoski, Pearson, Breslford, Ortegón y Chen, 1994), dificultades en tareas de emparejamiento intermodal consistentes en la elección de una expresión 
facial correspondiente a una vocalización no verbal (Hobson, Houston y Lee, 1988), incapacidad en la comprensión de términos emocionales (Hobson, Houston y Lee, 1989) o dificultades para asociar expresiones faciales a situaciones que habitualmente provocan emociones simples (Hobson, 1986a y 1986b).

Con relación a programas destinados a la enseñanza de habilidades sociales existen varios dirigidos a que las personas con TEA adquieran habilidades sociales e interpersonales, desarrollados en diferentes ambientes, tales como la escuela, la familia o contextos de terapia (Capadocia y Weiss, 2011; Mills y Marchant, 2011). Sin embargo, son escasos los estudios que examinan los programas diseñados para desarrollar lo que se refiere específicamente a las habilidades interpersonales como la capacidad de reaccionar a los propios problemas interpersonales y a los de los demás. Aunque diferentes estudios demostraron cómo los niños con TEA tienen dificultades en estos dominios (Buon, Dupoux, Jacob, Chaste, Leboyer y Zalla, 2013; Montgomery, Stoesz y McCrimmon, 2012) solo, destacamos un estudio que examinó el éxito de un programa de formación (Bonete, Molinero, Mata, Calero y Gómez-Pérez, 2016) que promovía el desarrollo de habilidades interpersonales de resolución de problemas sobre la base de un enfoque de formación por etapas.

Por otro lado, comprobamos que de los estudios referidos a la interiorización de los aprendizajes del alumnado con TEA con relación a la competencia emocional y social, podemos destacar el de Moreno, Aguilera, Saldaña y Álvarez (2005) quienes concluyen que los procesos educativos destinados a la mejora del reconocimiento de emociones, predicción de creencias y desarrollo social, Ilevados a cabo en situaciones de enseñanza reales y naturales, pueden repercutir en una generalización y extensión de estos aprendizajes escolares a otros contextos sociales. Por tanto, y desde el conocimiento que hemos recogido tras investigaciones previas en esta misma línea (Lozano, Alcaraz y Colás, 2010a y 2010b; Lozano y Alcaraz, 2012a y 2012b) estamos convencidos que es fundamental promover una enseñanza en la que colaboren docentes y familias, en contextos naturales, como resultan ser la escuela y el hogar, de modo tal que posibilite la adquisición de estas capacidades, con el fin de dotarlos de estrategias para vivir de un modo lo más normalizado posible. 
Materiales didácticos para la enseñanza de habilidades emocionales y sociales en alumnado con TEA: una investigación colaborativa en educación Primaria y Secundaria

josefina lozano Martínez, Maria del Carmen Cerezo Máiquez e Irina Sherezade Castillo

RECHE

\section{Colaboración Familia-Escuela}

La mayor parte de las investigaciones cuya temática es la familia, resaltan la importancia y el rol preponderante que ésta tiene como factor de soporte psicosocial (Benites, 2006). Pero, si tenemos en cuenta las dificultades que manifiestan las personas con TEA, es crucial que sus familias participen, de manera activa, en las diversas vertientes de su vida, incorporando en ellas el contexto escolar.

En esta misma línea Hortal (2011, p. 97) afirma que "si decimos, en general, que los padres son la mejor fuente de información sobre sus hijos y deberían ser los mejores aliados del centro educativo, no cabe ninguna duda de que, para estos niños, su colaboración resulta imprescindible". Por consiguiente, la colaboración y participación de los familiares de personas con TEA en los programas educativos fomenta el desarrollo del alumno, la generalización y reforzamiento de los aprendizajes conseguidos en un contexto determinado a otros entornos, facilitando, además, que esos aprendizajes sean más estables y duraderos en el tiempo (Tortosa, 2004). Este hecho hace que el hogar se convierta en uno de los contextos más favorecedores para el aprendizaje. En este sentido Ozonoff y Cathcart (1998) sugirieron que los programas de intervención que incluían el entorno del hogar en sus implementaciones eran eficaces para la mejora del desarrollo de los niños con TEA. En ese mismo enfoque Dunst, Bruder, Trivette, Hamby, Raab y McLean (2001) concluyeron que aquellas actividades de aprendizaje que se ofrecen a los niños en ambientes naturales tienen más probabilidades de estar asociadas a consecuencias positivas.

La coordinación y colaboración entre la familia, el centro educativo y los profesionales que ofertan una respuesta educativa al niño con este trastorno, debe ser muy estrecha para favorecer la implicación de la familia en el proceso educativo, estableciendo así las mismas pautas en casa y en el centro escolar (Lozano, 2010; Lozano y Alcaraz, 2011). Hemos de tener muy presente que, para sobrepasar los problemas frecuentes en la generalización de destrezas aprendidas en la escuela, es muy importante desarrollar programas con los padres para que las actividades de aprendizaje, experiencias y enfoques puedan ser utilizadas en el hogar y la comunidad desde la participación y el trabajo conjunto (Castro-Zubizarreta y García-Ruiz, 2016).

Por este motivo, Colás (2004), Colás y Contreras, (2013) indican que 
el buen desarrollo y progreso escolar depende, en gran medida, de la interiorización de significados, valores y prácticas que la institución escolar y familiar asuman en cada contexto sociocultural concreto. Partimos, por tanto, de que la colaboración y participación de los familiares de personas con TEA en los programas educativos fomenta el desarrollo del alumno, la generalización y reforzamiento de los aprendizajes conseguidos en un contexto determinado a otros entornos, facilitando, además, que esos aprendizajes sean más estables y duraderos en el tiempo (Tortosa, 2004).

\section{Marco empírico}

\section{Objetivos}

- Valorar el desarrollo de competencias emocionales del alumnado con TEA.

- Analizar la efectividad de un programa de intervención en el ámbito educativo y familiar para el desarrollo de competencias emocionales y sociales de alumnado con TEA.

- Comprobar los beneficios para el alumnado de la coordinación establecida entre familia y escuela.

\section{Participantes}

La muestra de estudio está compuesta por 6 alumnos de Educación Primaria y Secundaria, todos ellos presentan necesidades educativas especiales asociadas a TEA. Además, participaron en la investigación los docentes tutores de los alumnos, especialistas en audición y lenguaje y pedagogía terapéutica de dos centros de Educación Infantil y Primaria y de otro de Educación Secundaria de la Región de Murcia, las familias de los alumnos objeto de estudio, y el equipo de investigadores universitarios. Las características de los alumnos participantes en la investigación se resumen en la tabla 1, en la que los nombres de los alumnos son ficticios y no responden a la realidad con la finalidad de guardar la máxima confidencialidad. 
Materiales didácticos para la enseñanza de habilidades emocionales y sociales en alumnado con TEA: una investigación colaborativa en educación Primaria y Secundaria

Josefina lozano Martínez, Maria del Carmen Cerezo Máiquez e Irina Sherezade Castillo

RECHE

Tabla 1. Características de los alumnos participantes en la investigación.

\begin{tabular}{ccccccc}
\hline & Cristina & Fran & Andrea & Carlos & Raúl & Jaime \\
\hline Edad cronológica & 9 años & 10 años & 11 años & 11 años & 12 años & 17 años \\
\hline Escolarización & $\begin{array}{c}\text { Aula Ordi- } \\
\text { naria/Aula } \\
\text { Abierta }\end{array}$ & $\begin{array}{c}\text { Aula Or- } \\
\text { dinaria }\end{array}$ & $\begin{array}{c}\text { Aula Or- } \\
\text { dinaria }\end{array}$ & $\begin{array}{c}\text { Aula Or- } \\
\text { dinaria }\end{array}$ & $\begin{array}{c}\text { Aula Or- } \\
\text { dinaria }\end{array}$ & $\begin{array}{c}\text { Aula Abier- } \\
\text { ta/Aula } \\
\text { Ordinaria }\end{array}$ \\
\hline Nivel Educativo & $3^{\circ}$ de & $4^{\circ}$ de Edu- & $5^{\circ}$ de & $5^{\circ}$ de & $6^{\circ}$ de & $4^{\circ}$ de \\
& Educación \\
cación & Edu- & Edu- & Edu- & Educación \\
& Primaria & Primaria & cación & cación & cación & Secundaria \\
& TEA & TEA & TEA & TEA & TEA & TEA \\
\hline Diagnóstico & TEAmaria & Primaria & Primaria & \\
\hline
\end{tabular}

\section{Instrumentos de recogida de información}

- Cuestionario

Con el fin de valorar los posibles efectos que el proceso de enseñanzaaprendizaje de emociones y creencias ha podido tener sobre las habilidades emocionales y sociales del alumnado con TEA participante, se ha realizado a los alumnos un pretest y postest, que pretendía situar el punto de partida para comenzar el proceso de enseñanza-aprendizaje y contrastar los posibles avances tras el mismo. Para dicha evaluación se ha utilizado un cuestionario de valoración de habilidades emocionales y sociales (Lozano y Alcaraz, 2012) cumplimentado por los docentes y la familia de los alumnos objeto de estudio. Se trata de un cuestionario tipo Likert de 26 ítems, que se valoran del 1 al 5, donde 1 significa que la persona presenta dificultad o no tiene adquirida la capacidad que se evalúa, $y$, por el contrario, una puntuación de 5 nos indica que tiene adquirida dicha capacidad. Por lo tanto, cuanto mayor sea la puntuación, mayor será el grado de competencia emocional y social de la persona valorada. Este instrumento presenta un índice de fiabilidad de alfa de Cronback de .957 y se han establecido tres áreas de estudio: habilidades de capacidad intersubjetiva; básicas de relación social y de referencia conjunta.

- Seminarios

Se llevaron a cabo seminarios de trabajo con los docentes y familias de los distintos centros implicados, desarrollándose grupos de discusión para conversar y discutir sobre la problemática de estudio con el fin de que a través de sus discursos saliesen a la luz las diferentes subjetividades sobre la misma y se pudiese profundizar, por un lado, en la informa- 
Materiales didácticos para la enseñanza de habilidades emocionales y sociales en alumnado con TEA: una investigación colaborativa en educación Primaria y Secundaria Josefina lozano Martínez, Maria del Carmen Cerezo Máıquez e Irina Sherezade Castillo RECHE

ción más objetivada de los procesos y resultados obtenidos, y por otro, en propuestas de actuación para la mejora de la inclusión del alumnado con TEA en las aulas, así como el desarrollo de sus habilidades emocionales y sociales.

\section{Procedimiento}

El proceso de intervención educativa con los alumnos se llevó a cabo en el aula de referencia de los alumnos o en el hogar, dicho proceso se desarrolló durante un curso académico en el que se realizaban dos sesiones semanales de 45 minutos aproximadamente en cada contexto. Para la toma de decisiones en relación al proceso de enseñanza aprendizaje se creó un grupo de trabajo compuesto por docentes del centro educativo, las familias de los alumnos y los componentes del grupo de investigación universitario; estos últimos fueron guiando, a través de seminarios de acción-reflexión-acción, los distintos aspectos que componían el proceso de intervención, lo que permitía reflexionar sobre los avances y las dificultades surgidas. El equipo universitario destacó su labor en el diseño de materiales didácticos y orientación del proyecto, mientras que docentes y familias han realizado el trabajo diario con estos alumnos en contextos naturales como son la escuela o el hogar; guiando a sus alumnos/hijos en la realización de actividades, interviniendo de forma estructurada y sistemática, incluyendo preguntas, feedback y explicación o elogio, y a partir de ahí, llegando a proponer experiencias de la vida cotidiana del alumno para conseguir la extrapolación de los aprendizajes y su posible generalización.

\section{Materiales didácticos}

Para la enseñanza de competencias emocionales se utilizaron las colecciones de materiales didácticos tituladas "Aprende con Zapo. Propuestas didácticas para el aprendizaje de habilidades emocionales y sociales" (Lozano y Alcaraz, 2009) e "Ilusiónate con Tachín y sus historias emocionales y sociales" (Lozano, Cerezo, Merino y Castillo, 2014). Los docentes del centro trabajaron fundamentalmente con los materiales de la primera colección y empezaron a introducir otros materiales complementarios de la segunda. En el ámbito familiar se utilizaron únicamente estas historias sociales y emocionales debido a las posibilidades que 
Materiales didácticos para la enseñanza de habilidades emocionales y sociales en alumnado con TEA: una investigación colaborativa en educación Primaria y Secundaria

josefina lozano Martínez, Maria del Carmen Cerezo Máiquez e Irina Sherezade Castillo

RECHE

ofrecían al ser unos materiales didácticos que contemplaban escenarios naturales y situaciones que pueden surgir para los alumnos en el centro, en el contexto familiar o en su contexto social cercano, y que habían sido creadas desde las dificultades que estas personas con TEA presentaban desde las opiniones que los docentes y las familias exponían en los seminarios de trabajo.

La colección "Aprende con Zapo", es un recurso didáctico que incorpora una serie de actividades para la intervención educativa en esta área, proponiendo en sus diferentes niveles, estructurados de menor a mayor complejidad, una secuenciación de tareas que pueden ser utilizadas al mismo tiempo para la evaluación y la enseñanza. Dichas tareas comienzan con la identificación de emociones básicas y complejas a través de las expresiones faciales en dibujos y fotografías, continúan con el reconocimiento y atribución causal de emociones a situaciones concretas y finalizan con la comprensión de que las personas pueden tener distintos modos de percepción, conocimiento y creencia, tal y como se muestra en el anexo 1. Todos estos niveles incluyen una gran variedad de actividades, personajes y contextos con la finalidad de que el material se adapte lo máximo posible a las necesidades y demandas de los alumnos con TEA. Las actividades se presentan tanto en formato impreso como en formato digital.

Por otro lado, las historias sociales de la colección "Ilusiónate con Tachín y sus historias emocionales y sociales" (Lozano, Cerezo, Merino y Castillo, 2014) son materiales didácticos creados para el proceso de enseñanza-aprendizaje. Estos materiales contemplan situaciones que pueden surgir para los alumnos en escenarios naturales como el centro escolar, el contexto familiar o su contexto social cercano. Recogen pautas a seguir para la resolución de pequeños conflictos y los personajes de dichas historias pueden sentir tanto emociones básicas (alegría, tristeza, enfado y miedo) como complejas (interés, aburrimiento, sorpresa y pensamiento) a lo largo de las distintas situaciones que se desarrollan en la historia. Asimismo, incorporan aquellas tareas que se han observado que resultan de gran dificultad para el alumnado a través de la implementación del material referido anteriormente, como son la enseñanza de creencias (sobre todo la creencia falsa y predicción de la acción) y las situaciones de comunicación metafórica (mentira, mentira piadosa e ironía). Para favorecer la extrapolación y personalización de las situaciones a las personas con TEA, estos materiales incluyen preguntas que hacen 
referencia a cómo los alumnos viven o han vivido situaciones semejantes a la que la historia social presenta. Ejemplos de las distintas tareas de dichos materiales aparecen reflejados en el anexo 2.

\section{Resultados}

Los resultados obtenidos en el cuestionario valorativo de habilidades emocionales y sociales y en la resolución de tareas de los materiales didácticos, muestran que todos los alumnos participantes en la investigación obtuvieron mayores puntuaciones tras el proceso de enseñanzaaprendizaje. En las Figuras 1 y 2 observamos el avance reflejado en las puntaciones del cuestionario valorativo realizado por los docentes y las familias:

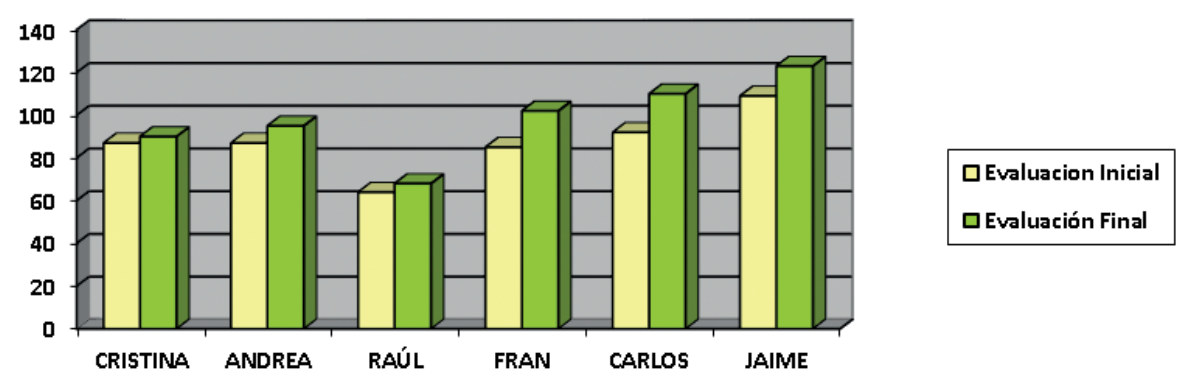

Figura 1. Puntuaciones obtenidas en el cuestionario valorativo de habilidades emocionales y sociales según los docentes.

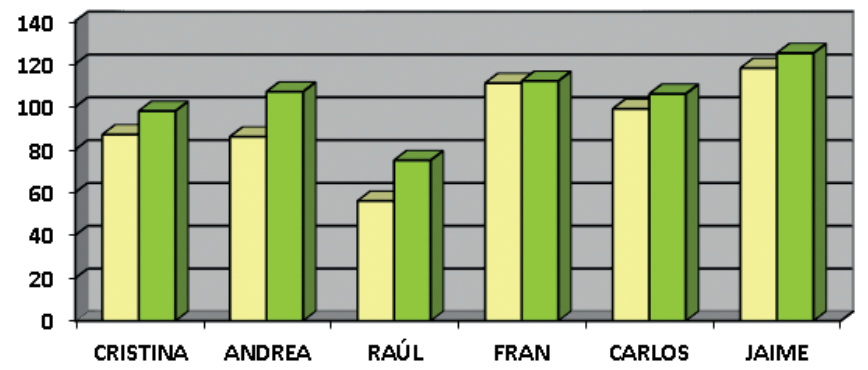

Figura 2. Puntuaciones obtenidas en el cuestionario valorativo de habilidades emocionales y sociales según las familias. 
Si estudiamos de forma individualizada los progresos de cada uno de los participantes, teniendo en cuenta los resultados del cuestionario, de los materiales utilizados para la evaluación de comprensión de emociones y las valoraciones de los padres verbalizadas en los seminarios de trabajo conjunto llevados a cabo entre docentes, familiares y equipo universitario, cabe destacar lo siguiente para cada uno de los casos:

\section{Cristina}

Si describimos de forma detallada los progresos de la participante, se comprueba que, bajo la visión de la familia, la alumna ha mejorado sobre todo sus habilidades de capacidad intersubjetiva ya que muestra mayor interés ante las emociones de los demás, por lo que te mira o te pregunta en un mayor número de ocasiones cuando alguien muestra emociones tanto básicas como complejas y se aprecian valores más altos en su capacidad para reconocer los estados mentales propios y ajenos asociándolos a situaciones concretas, respondiendo ante ellos y justificándolos en un mayor número de ocasiones. Además, se observa mayor espontaneidad en la expresión de emociones sobre todo alegría y enfado.

Para el "Área de Habilidades de Referencia Conjunta", tras el proceso de intervención educativa, obtuvo mayor valoración por parte del docente en sus habilidades para compartir sonrisas, además mejoró su habilidad para compartir emociones con los demás, mostrándose más interesada y preocupada por los gestos expresivos de otra persona. Los docentes destacan también un aumento en las ocasiones en las que la alumna mira lo que los demás hacen sobre los objetos.

Finalmente, para el "Área de Habilidades Básicas de Relación Social", la alumna mejoró sus competencias y motivación para relacionarse con los demás, quedando reflejado en la capacidad para pedir cooperación ante un grupo. Esta situación también es manifestada por los docentes. Es capaz de resolver adecuadamente los conflictos que aparecen en las historias sociales, aunque presenta dificultad ante las mentiras piadosas.

\section{Andrea}

Con relación al "Área de Habilidades Básicas de Relación Social" la alumna mejoró su capacidad para relacionarse e interactuar con otros; 
favoreciendo su espontaneidad, frecuencia y calidad, dado que la familia resalta avances, sobre todo, al pedir cooperación ante un grupo de niños que están jugando.

Para el "Área de Habilidades de Referencia Conjunta", tras el proceso de intervención educativa, obtuvo mayor valoración por parte de la familia en su capacidad para prestar, atención e interés a los actos de los demás desarrollados sobre objetos, llegando incluso a imitar en un mayor número de ocasiones. Además, mejoró su habilidad, bajo la perspectiva de la familia y el docente, para compartir emociones con los demás, mostrándose más interesada y preocupada por los gestos expresivos y emociones de otras personas; dicho avance se muestra sobre todo en las emociones complejas.

En cuanto al "Área de Habilidades de Capacidad Intersubjetiva", se aprecian valores más altos en su capacidad para reconocer los estados mentales propios y ajenos, asociándolos a situaciones concretas y respondiendo al porqué de dicha situación (dicho avance se identifica en las ocasiones en las que la emoción generada es compleja); todo ello hizo que la alumna utilizara en un mayor número de ocasiones términos emocionales refiriéndose a ella y a otros.

Además, obtuvo un mayor rendimiento, según la familia, en la comprensión de creencias, en la resolución de conflictos que aparecían en las historias y en la generalización de lo aprendido de un contexto a otro.

\section{Raúl}

Bajo la visión de la familia, el alumno ha mejorado sobre todo sus habilidades de capacidad intersubjetiva ya que muestra mayor interés ante las emociones de los demás por lo que te mira o te pregunta en un mayor número de ocasiones cuando alguien muestra emociones tanto básicas como complejas y se aprecian valores más altos en su capacidad para reconocer los estados mentales propios y ajenos asociándolos a situaciones concretas, respondiendo ante ellos y justificándolos en un mayor número de ocasiones. Además, se observa mayor espontaneidad en la expresión de emociones de forma general según la familia y cuando identifica que va a conseguir o no lo que quiere según los docentes.

Para el "Área de Habilidades de Referencia Conjunta", tras el proceso de intervención educativa, obtuvo mayor valoración por parte del docente en sus habilidades para compartir emociones con los demás, 
Materiales didácticos para la enseñanza de habilidades emocionales y sociales en alumnado con TEA: una investigación colaborativa en educación Primaria y Secundaria

josefina lozano Martínez, Maria del Carmen Cerezo Máiquez e Irina Sherezade Castillo

RECHE

mostrándose más interesado y preocupado por los gestos expresivos de otra persona.

La familia destaca también un aumento en las ocasiones en las que el alumno mira lo que los demás hacen sobre los objetos y la realización de juego simbólico.

Todos los avances anteriores dan lugar a un aumento en el empleo de términos emocionales; avance identificado tanto por docentes como por familia.

\section{Fran}

El alumno ha mejorado en mayor medida, sus Habilidades de Capacidad Intersubjetiva ya que muestra mayor interés ante las emociones de los demás por lo que te mira o te pregunta en un mayor número de ocasiones cuando alguien muestra emociones tanto básicas como complejas y se aprecian valores más altos en su capacidad para reconocer los estados mentales propios y ajenos asociándolos a situaciones concretas, respondiendo ante ellos y justificándolos en un mayor número de ocasiones. Además, se observa mayor espontaneidad en la expresión de emociones sobre todo alegría y enfado.

Para el "Área de Habilidades de Referencia Conjunta", tras el proceso de intervención educativa, obtuvo mayor valoración por parte del docente en sus habilidades para compartir sonrisas, además mejoró su habilidad para compartir emociones con los demás, mostrándose más interesado y preocupado por los gestos expresivos de otra persona.

Finalmente, para el "Área de Habilidades Básicas" de relación social, mejoró sus competencias y motivación para relacionarse con los demás, quedando reflejado en la capacidad para pedir cooperación ante un grupo. Asimismo, tras el proceso, es capaz de realizar juego simbólico.

En el desarrollo de las historias sociales comprobamos que es capaz de generalizar los aprendizajes de un contexto a otro.

\section{Carlos}

El alumno muestra más regulación en sus emociones sobre todo en el control de la tristeza y el enfado, va asumiendo la consecuencia de sus actos y se interesa más por las emociones de los demás. Se enfada, pero 
con menos intensidad y frecuencia además de razonar más sobre las distintas situaciones.

Reconoce de forma autónoma todas las emociones, aunque confunde solo la de tristeza, se muestra más maduro e independiente en sus tareas y organización de casa y escolar.

Ha dejado de morderse los dedos, mantiene buenas relaciones dentro del colegio y con la familia. Verbaliza y pide mayor autonomía y confianza para desarrollar sus responsabilidades.

\section{Jaime}

Tras el proceso de intervención educativa, Jaime mejoró sobre todo en el "Área de Habilidades de Capacidad Intersubjetiva", pues se muestra más interesado y preocupado por los gestos expresivos y emociones de otras personas. Se aprecian también valores más altos en su capacidad para reconocer los estados mentales propios y ajenos, asociándolos a situaciones concretas y respondiendo al porqué de dicha situación, Ilegando incluso a personalizar la situación del protagonista de la historia y a generalizar en el contexto familiar lo trabajado en el centro educativo. Igualmente obtuvo un mayor rendimiento en la comprensión de que los deseos y creencias pueden causar emociones llegando a identificar qué emociones pueden causar. Así mismo, utiliza en un mayor número de ocasiones términos emocionales refiriéndose a él y a otros. Además, obtuvo un mayor rendimiento, según ambos informantes, en la comprensión de creencias, en la resolución de conflictos de las historias, y en los juicios de emoción basados en comunicación metafórica.

\section{Discusión y conclusiones}

En primer lugar si nos centramos en el primer y segundo objetivo, valorar el desarrollo de competencias emocionales y sociales del alumnado con TEA y valorar la efectividad de un programa de intervención en el ámbito educativo y familiar para el desarrollo de competencias emocionales y sociales de alumnado con TEA, hemos de destacar el avance que ha supuesto para cada uno de los sujetos estudiados, así como la importancia del trabajo de las competencias mencionadas a través de los materiales seleccionados, tanto en formato impreso como digital, de Aprende con 
Materiales didácticos para la enseñanza de habilidades emocionales y sociales en alumnado con TEA: una investigación colaborativa en educación Primaria y Secundaria

Josefina lozano Martínez, Maria del Carmen Cerezo Málquez e Irina Sherezade Castillo

RECHE

Zapo. Propuestas didácticas para el aprendizaje de habilidades emocionales y sociales (Lozano y Alcaraz, 2009), y del material de Ilusiónate con Tachín y sus historias emocionales y sociales (Lozano et al, 2014) más aquellas dinámicas, que se han generado en el centro educativo y en el hogar, y que han favorecido un proceso de enseñanza-aprendizaje en contextos naturales que ha resultado dinámico y complementario, en la medida que cada miembro participante ha culminado, desde su contexto el desarrollo y la generalización de lo iniciado en otro. De este modo, se han experimentado numerosos avances en los alumnos objeto de estudio, en cuanto al desarrollo de determinadas competencias (emocionales y sociales) como hemos podido precisar en los resultados presentados para cada alumno, lo que ha supuesto el comienzo de la generalización de los aprendizajes de un contexto a otro.

Si nos centramos en el objetivo relacionado con la coordinación del trabajo conjunto entre escuela y la familia para el desarrollo de competencias emocionales y sociales en alumnado con TEA de educación primaria y secundaria a través de materiales procedentes de varias propuestas didácticas concretas en formato impreso y digital, tenemos que considerar que el resultado ha sido satisfactorio, puesto que hemos encontrado un alto grado de implicación desde los ámbitos de trabajo (familia y escuela) y se han desarrollado las actividades propuestas en dos formatos (impreso y digital), proporcionado resultados positivos, generalizables en algunos casos de un contexto a otro en cuanto al desarrollo de habilidades emocionales y sociales en los participantes. Los resultados obtenidos del desarrollo de los distintos seminarios llevados a cabo, de las observaciones de campo, nos dan información suficiente para valorar el proceso llevado a cabo en cada contexto, el reparto de tareas por cada miembro participante y los beneficios de un trabajo diseñado para ser trabajado en contextos naturales, donde las investigaciones precedentes (Dunst, Bruder, Trivette, Hamby, Raab y McLean,2001; Ozonoff y Cathcart, 1998; Moreno, Aguilera, Saldaña y Álvarez, 2005) informaban de dichos beneficios.

Por tanto, y desde la prudencia que nos permiten los resultados de esta investigación, es aconsejable que los métodos educativos que se sigan con este alumnado se dirijan hacia la generalización de los aprendizajes implicando a todos los agentes educativos que participan, democráticamente, en las decisiones que le atañen: profesorado, familia y otros profesionales, dado que se ha observado que este procedimiento, al igual que planteaban Castro-Zubizarreta y García-Ruiz (2016), Tortosa 
Materiales didácticos para la enseñanza de habilidades emocionales y sociales en alumnado con TEA: una investigación colaborativa en educación Primaria y Secundaria Josefina lozano Martínez, Maria del Carmen Cerezo Málquez e Irina Sherezade Castillo RECHE

(2004) y Hortal (2011), fomenta el desarrollo del alumno, la generalización y reforzamiento de los aprendizajes conseguidos en un contexto determinado a otros entornos.

\section{Referencias bibliográficas}

American Psychiatric Association (2013). DSM-5 Development. Recuperado de http:// www.dsm5.org/

American Psychiatric Association (2014). Actualización de la codificación del DSM-5. Suplemento del Manual Diagnóstico y Estadístico de Trastornos Mentales, Quinta Edición. Arlington, VA: American Psychiatric Association. Recuperado de http://psychiatryonline.org/pb/assets/raw/dsm/pdf/Spanish_DSM5\%20Coding\%20Update_Final.pdf

Bauminger, N. (2007). Brief report: individual social-multi-modal intervention for HFASD. Journal of Autism and Developmental Disorders, 37(8), 1593-1604. doi: 10.1007/ s10803-006-0245-4.

Benites, L. (2006). Familia y asertividad en adolescentes que asisten a centros comunales. Avances en Psicología, 14(1), 47-72.

Bernard-Ripoll, S. (2007). Using a self-as-model video combined with social stories to help a child with asperger syndrome understand emotions. Focus on Autism and Other Developmental Disabilities, 22(2), 100-106.

Blacher, J. y Howell, E. (2007). Unlocking the mystery of social deficits in autism: theory of mind as key. Except Parent, 37(8), 96-98.

Bonete, S., Molinero, M., Mata, M., Calero, M. D. y Gómez-Pérez, M. M. (2016). Effectiveness of Manualised Interpersonal Problem-Solving Skills Intervention for Children with Autism Spectrum Disorder (ASD). Psicothema, 28(3), 304-310. doi: 10.7334/ psicothema2015.206.

Buon, M., Dupoux, E., Jacob, P., Chaste, P., Leboyer, M. y Zalla, T. (2013). The role of causal and intentional judgments in moral reasoning in individuals with high functioning autism. Journal of Autism and Development Disorders, 43, 458-470. doi:10.1007/ s10803-012-1588-7.

Cappadocia, M. C. y Weiss, J. A. (2011). Review of social skills training groups for youth with Asperger syndrome and high functioning autism. Research in Autism Spectrum Disorders, 5(1), 70-78.

Castro-Zubizarreta, A. y García-Ruiz, R. (2016). Vínculos entre familia y escuela: visión de los maestros en formación. Magis: Revista Internacional de Investigación en Educación, 9(18), 193-208.

Colás, M. P. (2004). La construcción de una pedagogía de género para la igualdad, en Rebollo A. y Mercado, I. (Coords.). Mujeres y desarrollo en el siglo XXI: Voces para la igualdad. Madrid: Mcgraw-Hill.

Colás, P. y Contreras, J.A. (2013). La participación de las familias en los centros de educación primaria. Revista de Investigación Educativa, 31(2), 485-499.

Dunst, C.J., Bruder, M.B., Trivette, C.M., Hamby, D., Raab, M. y Mclean, M. (2001). Cha- 
Materiales didácticos para la enseñanza de habilidades emocionales y sociales en alumnado con TEA: una investigación colaborativa en educación Primaria y Secundaria Josefina lozano Martínez, Maria del Carmen Cerezo Málquez e Irina Sherezade Castillo

RECHE

racteristics and consequences of everyday natural learning opportunities. Topics in Early Childhood Special Education, 21(2), 68-92.

García-Gómez, A., Risco, M., Rubio, J.C., Guerrero, E. y García-Peña, I.M. (2014). Efectos de un Programa de Equitación Adaptada y Terapéutica en un Grupo de Niños con Trastornos del Espectro Autista. Electronic Journal of Research in Educational Psychology, 12(1), 107-128.

Golan, O. y Baron-Cohen, S. (2006). Systemizing empathy: Teaching adults with Asperger syndrome or high-functioning autism to recognize complex emotions using interactive multimedia. Development and Psychopathology, 18, 591-617.

Golan, O., Ashwim, E., Granader, Y., Mcclintock, S., Day, K., Leggett, V. y Baron-Cohen, S. (2010). Enchancing emotion recognition in children with autism spectrum conditions: an intervention using animated vehicles with real emotional faces. Journal of Autism and Developmental Disorders, 40(3), 269-279. doi: 10.1007/s10803-009-0862-9.

Hadwin, J., Baron-Cohen, S., Howlin, P. y Hill, K. (1996). Can we teach children with autism to understand emotions, belief or pretende? Development and Psychopatho$\log , 8(2), 345-365$.

Hobson, P. (1986a). The autistic child's appraisal of expressions of emotion. Journal of Child Psychology and Psychiatry, 27(3), 321-342.

Hobson, P. (1986b). The autistic child's appraisal of expressions of emotion: a further study. Journal of Child Psychology and Psychiatry, 27(5), 671-680.

Hobson, P., Houston, J. y Lee, A. (1988). Emotion recognition in autism: Coordinating faces and voices. Psychological Medicine, 18, 911-923.

Hobson, P., Houston, J. y Lee, A. (1989). Recognition of emotion by mentally retarded adolescents and young adults. American Journal on Mental Retardation, 93, 434-443.

Hortal, C. (Coord.) (2011). Alumnado con Trastorno del Espectro Autista. Barcelona: Editorial GRAÓ.

Howlin, P. (2008). ¿Se puede ayudar a los niños con trastornos del espectro autista a adquirir una "teoría de la mente"? Logopedia, Foniatría y Audiología, 28(2), 74-89.

Kanner, L. (1943). Autistic disturbance of affective contact. Nervous Child, 2, 107-134.

Llevot, N. y Bernad, O. (2015). La participación de las familias en la escuela: factores clave. Revista de la Asociación de Sociología de la Educación, 8(1), 57-70. Recuperado de http://www.ase.es/rase/index.php/RASE/article/view/370/371

Loveland, K., Tunali-Kotoski, B., Pearson, D., Breslford, K., Ortegón, J., y Chen, R. (1994). Imitation and expression of facial affect in autism. Development and Psychopathology, 6, 433-444.

Lozano, J. y Alcaraz, S. (2009). Aprende con Zapo. Propuestas didácticas para el aprendizaje de habilidades emocionales y sociales. Madrid: Wolters Kluwer España.

Lozano, J. y Alcaraz, S. (2010). Enseñar emociones para beneficiar las habilidades sociales de alumnado con trastornos del espectro autista. Educatio Siglo XXI, 28(2), 261288.

Lozano, J. y Alcaraz, S. (2011). La investigación colaborativa como mejora de los procesos de enseñanza de competencias emocionales del alumnado con trastornos del espectro autista. En J. Maquilón; A. Mirete; A. Escarbajal y A. M. Jiménez. Cambios 
Materiales didácticos para la enseñanza de habilidades emocionales y sociales en alumnado con TEA: una investigación colaborativa en educación Primaria y Secundaria Josefina lozano Martínez, Maria del Carmen Cerezo Málquez e Irina Sherezade Castillo RECHE

educativos y formativos para el desarrollo humano y sostenible. pp:213, 223 Universidad de Murcia. Servicio de publicaciones.

Lozano, J. y Alcaraz, S. (2012a). Respuesta educativa al alumnado con trastornos del espectro autista. Madrid: La Muralla.

Lozano, J. y Alcaraz, S. (2012b). Enseñanza de emociones y creencias en alumnos con trastornos del espectro autista: efectos sobre las habilidades sociales cotidianas. Revista de educación, 358, 357-381.

Lozano, J.(Coord.), Cerezo, M.C., Merino, S. y Castillo, I. (2014). Ilusiónate con Tachin y sus historias emocionales y sociales. Recuperado de http://hdl.handle. net/10201/39773.

Lozano, J., Alcaraz, S. y Colás, M. P. (2010a). Experiencia educativa sobre la comprensión de emociones y creencias en alumnado con trastornos del espectro autista. Revista de Investigación Educativa, 28(1), 65-78.

Lozano, J., Alcaraz, S. y Colás, P. (2010b). La enseñanza de emociones y creencias a alumnos con trastornos del espectro autista: una investigación colaborativa. Profesorado, 14(1), 367-382.

Mills, R. y Marchant, S. (2011). Intervention in autism: A brief review of the literature. Tizard Learning Disability Review, 16(4), 20-35.

Montgomery, J. M., Stoesz, B. M. y Mccrimmon, A. W. (2012). Emotional intelligence, theory of mind, and executive functions as predictors of social outcomes in young adults with Asperger Syndrome. Focus of Autism and Other Developmental Disabilities, 28(1), 4-13.

Moreno, F. J., Aguilera, A., Saldaña, D. y Álvarez, R. (2005). ¿Cómo mejorar la educación del alumnado con autismo? Una propuesta desde el sistema escolar sevillano. Apuntes de Psicología, 23(3), 257-374.

Ojea, M. (2010). Emociones en niños y niñas autistas: programa de desarrollo de la comprensión social (1a.ed.). Valencia: PSYLICOM Ediciones.

Ozonoff, S. y Cathcart, K. (1998). Effectiveness of a home program intervention for young childrenwith autism. Journal of Autism and Developmental Disorders, 28, 25-32.

Rivière, A. (2002). IDEA: Inventario de espectro autista. Buenos Aires: Fundec.

Tanaka, J.W., Wolf, J.M., Klaiman, C., Koenig, K., Cockburn, J., Herlihy, L. y Schultz. R.T. (2010). Using computerized games to teach face recognition skills to children with autism spectrum disorder: The Let's Face It! Program. Journal of Child Psychology and Psychiatry, 51(8), 944-952. doi:10.1111/j.1469-7610.2010.02258.

Tortosa, F. (2004). Intervención educativa en el alumnado con trastornos del espectro autista. Recuperado de http://www.psie.cop.es/uploads/murcia/Intervención\%20TEA. pdf

Wing, L. (1988). The continuum of autistic characteristics. En E. Schopler y G.B. Mesibov (Eds.), Diagnosis and Assessment in Autism. New York: Plenum. 
Materiales didácticos para la enseñanza de habilidades emocionales y sociales en alumnado con TEA: una investigación colaborativa en educación Primaria y Secundaria

Josefina lozano Martínez, Maria del Carmen Cerezo Máiquez e Irina Sherezade Castillo

\section{Anexos}

Anexo 1: Estructura del material didáctico "Aprende con Zapo. Propuestas didácticas para el aprendizaje de habilidades emocionales y sociales" (Lozano y Alcaraz, 2009).

\section{EMOCIONES BÁSICAS Y COGNITIVAS}

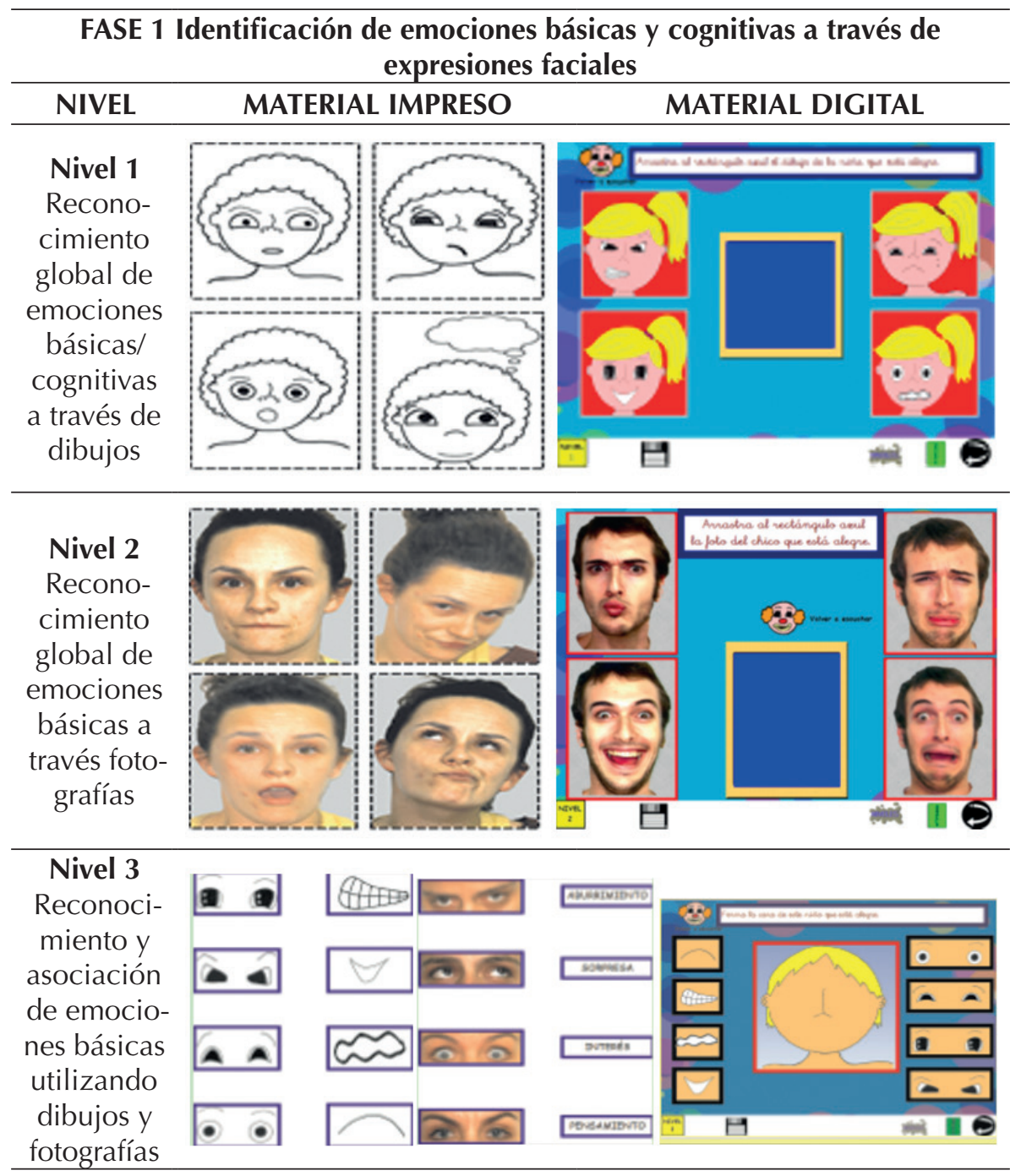


Materiales didácticos para la enseñanza de habilidades emocionales y sociales en alumnado con TEA: una investigación colaborativa en educación Primaria y Secundaria Josefina lozano Martínez, Maria del Carmen Cerezo Málquez e Irina Sherezade Castillo RECHE

\section{FASE 2 Reconocimiento y atribución causal de emociones a través de historias}

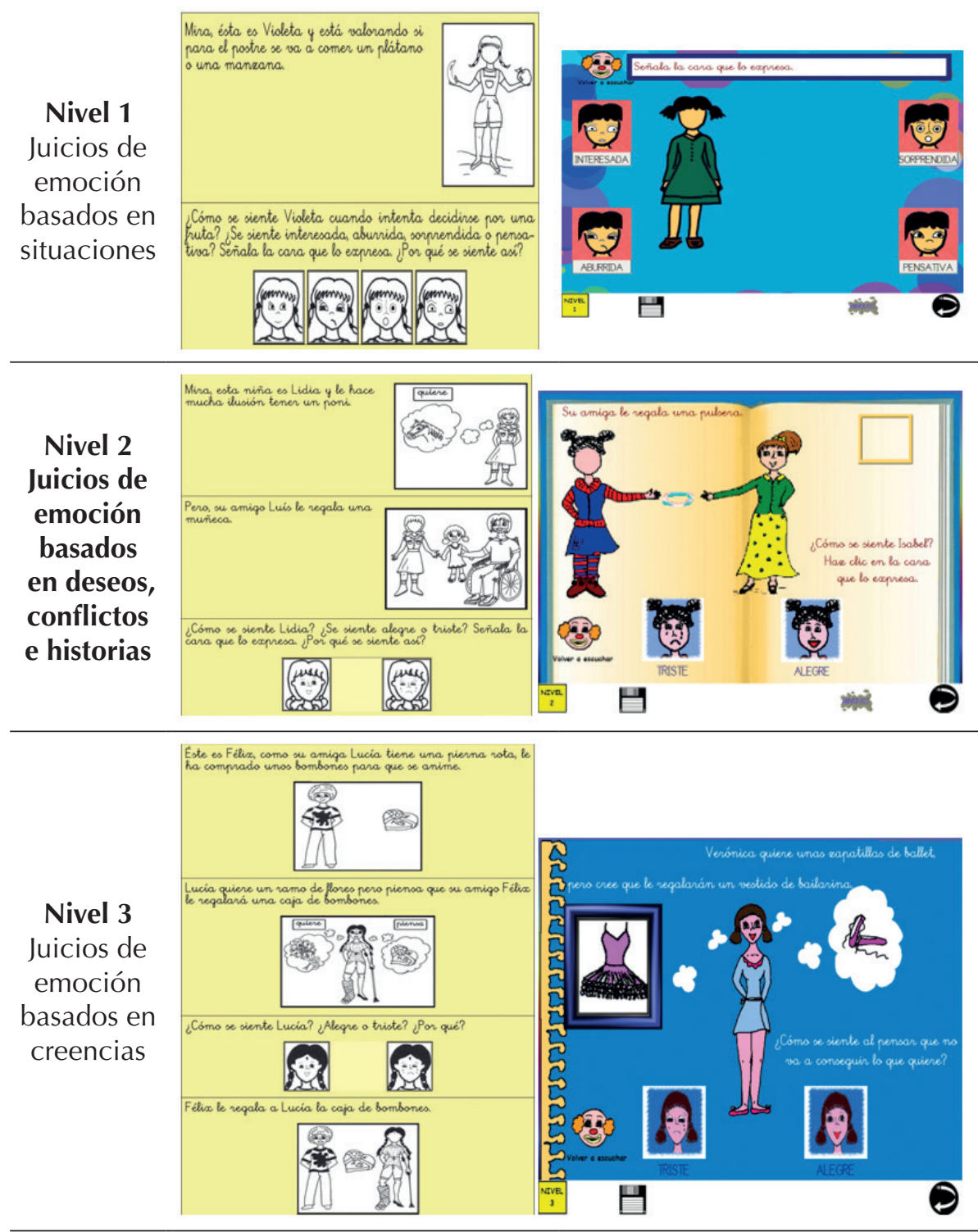


Materiales didácticos para la enseñanza de habilidades emocionales y sociales en alumnado con TEA: una investigación colaborativa en educación Primaria y Secundaria

Josefina lozano Martínez, Maria del Carmen Cerezo Máiquez e Irina Sherezade Castillo RECHE

\section{Nivel 4 \\ Juicios de \\ emoción \\ basados \\ en comu- \\ nicación \\ metafórica}

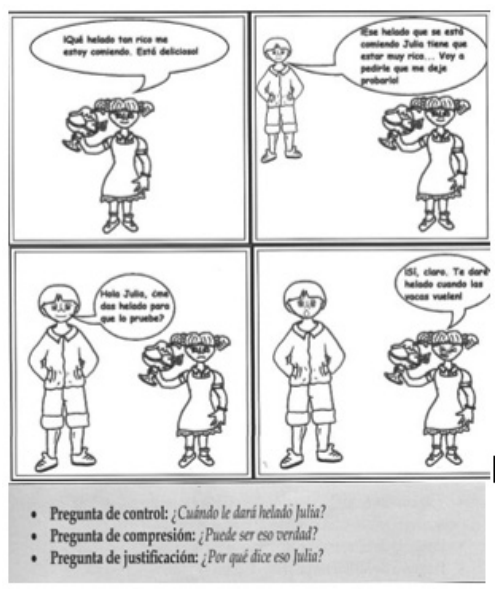

Nivel 5

Creación

de histo-

rias emo-

cionales

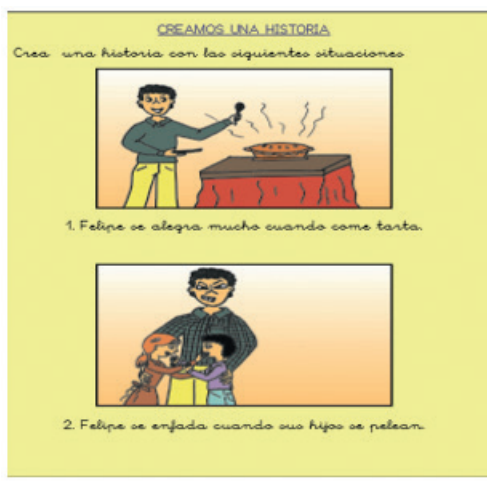

\section{ENSEÑANZA DE CREENCIAS}

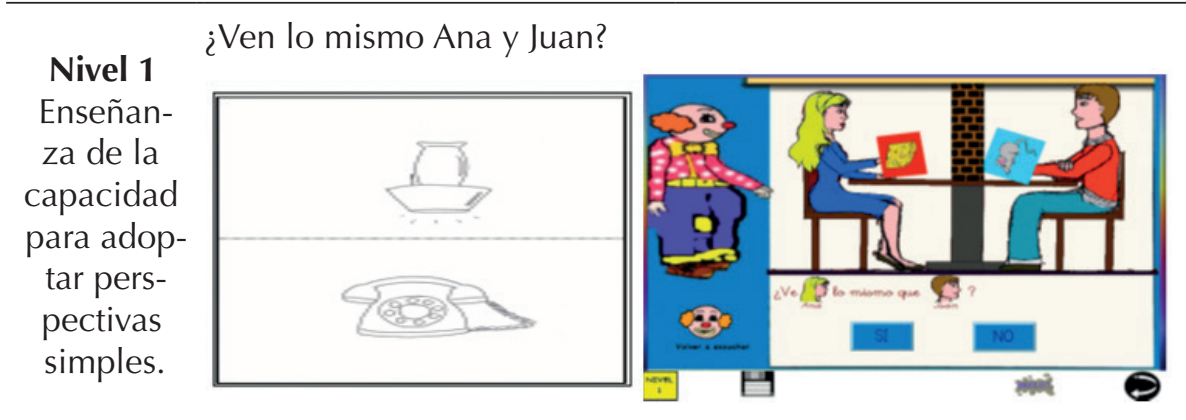


Materiales didácticos para la enseñanza de habilidades emocionales y sociales en alumnado con TEA: una investigación colaborativa en educación Primaria y Secundaria Josefina lozano Martínez, Maria del Carmen Cerezo Málquez e Irina Sherezade Castillo RECHE

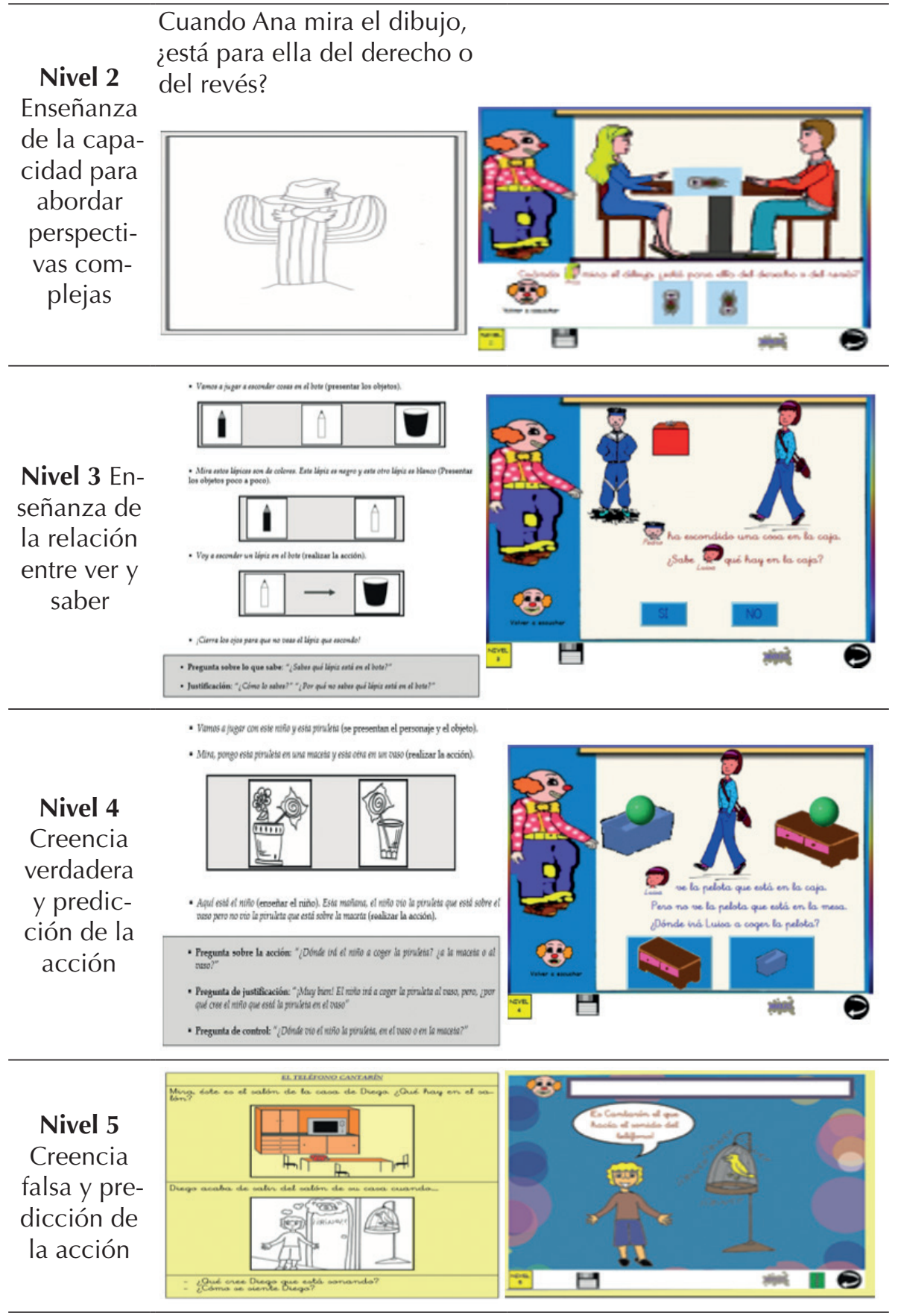


Materiales didácticos para la enseñanza de habilidades emocionales y sociales en alumnado con TEA: una investigación colaborativa en educación Primaria y Secundaria

Josefina lozano Martínez, Maria del Carmen Cerezo Máiquez e Irina Sherezade Castillo

Anexo 2: Estructura del material didáctico "Ilusiónate con Tachín y sus historias emocionales y sociales" (Lozano, Cerezo, Merino y Castillo, 2014).

\section{HISTORIAS EMOCIONALES Y SOCIALES}

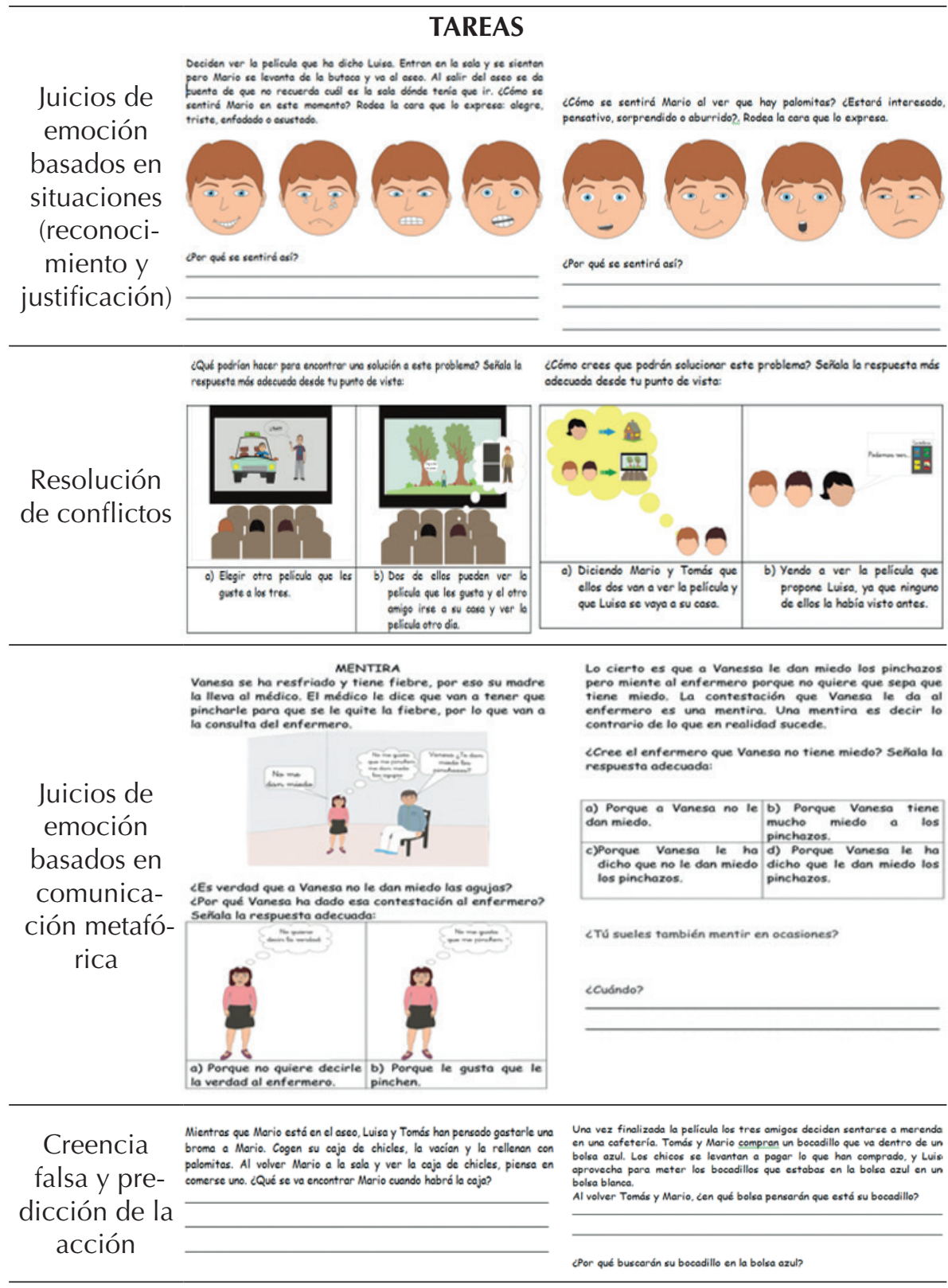


Materiales didácticos para la enseñanza de habilidades emocionales y sociales en alumnado con TEA: una investigación colaborativa en educación Primaria y Secundaria Josefina lozano Martínez, Maria del Carmen Cerezo Málquez e Irina Sherezade Castillo RECHE

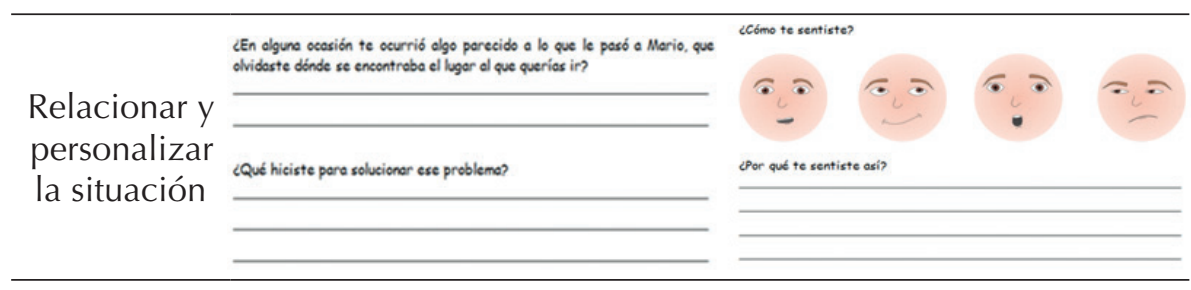

